\title{
Agent Affecting Nervous System
}

National Cancer Institute

\section{Source}

National Cancer Institute. Agent Affecting Nervous System. NCI Thesaurus. Code C78272.

An agent that exerts a physiologic effect on neurotransmission or on any anatomic entity that is part of the nervous system, including the central nervous system (CNS) and peripheral nervous system (PNS). 